\title{
Iodinated Contrast Encephalopathy
}

\author{
Ke Chen, Sami Obaid, Laurent Guillon-Létourneau, Dang Khoa Nguyen
}

Keywords: Angiography, Wada test, Contrast encephalopathy

doi:10.1017/cjn.2017.24

Can J Neurol Sci. 2017; 44: 432-433

A 39-year-old, left-handed woman with right temporal lobe epilepsy was admitted for an intracarotid sodium amobarbital procedure (Wada test) to establish language and memory representation of each hemisphere and hence determine if she was suitable for a right anterior temporal lobectomy. This procedure involves catheterization of a carotid artery in a first session (and the other in another session) via the femoral artery before amobarbital injection and, therefore, includes an angiogram of the carotid vasculature using a contrast agent. Following left intracarotid injection of iopamidol contrast agent, she manifested a rapid decrease in consciousness and progressive right hemiplegia, requiring intubation and admission to the intensive care unit. Continuous electroencephalogram monitoring showed severe left hemispheric slowing. Initial brain magnetic resonance imaging (MRI) showed left hemispheric edema suggestive of iodinated contrast encephalopathy (Figure 1). Her level of consciousness and motor strength markedly improved within 2 weeks and neuropsychological deficits resolved over the following months. A repeat neuropsychological evaluation 15 months later showed significant improvement of cognitive function, albeit with some mild language deficits and psychomotor slowing. Serial followups over the next 2 years revealed gradual regression of the MRI abnormalities (Figure 2).

Iodinated contrast encephalopathy is an uncommon, usually self-limiting and reversible complication after angiography. ${ }^{1-4}$ The mechanism of neurotoxicity is thought to be a temporary disruption of the blood-brain barrier by the iodinated contrast and parenchymal inflammation., ${ }^{2,3}$ The most frequent clinical presentations are transient cortical blindness, seizures, cognitive dysfunctions, and focal neurological deficits including sensorimotor impairments. ${ }^{2}$ Although severe and long-lasting in our patient, most reported cases have lasted less than 2 to 3 hours. ${ }^{1}$ Risk factors for iodinated contrast encephalopathy include hypertension and renal failure. ${ }^{2,3}$ Common neuroimaging findings are contrast enhancement and oedema of the cortex, which can be found in up to $54 \%$ of computed tomography scans performed within 2 hours of the event. ${ }^{2}$ Brain MRI scans more frequently reveal a pattern of focal or diffuse cortical T2-weighted
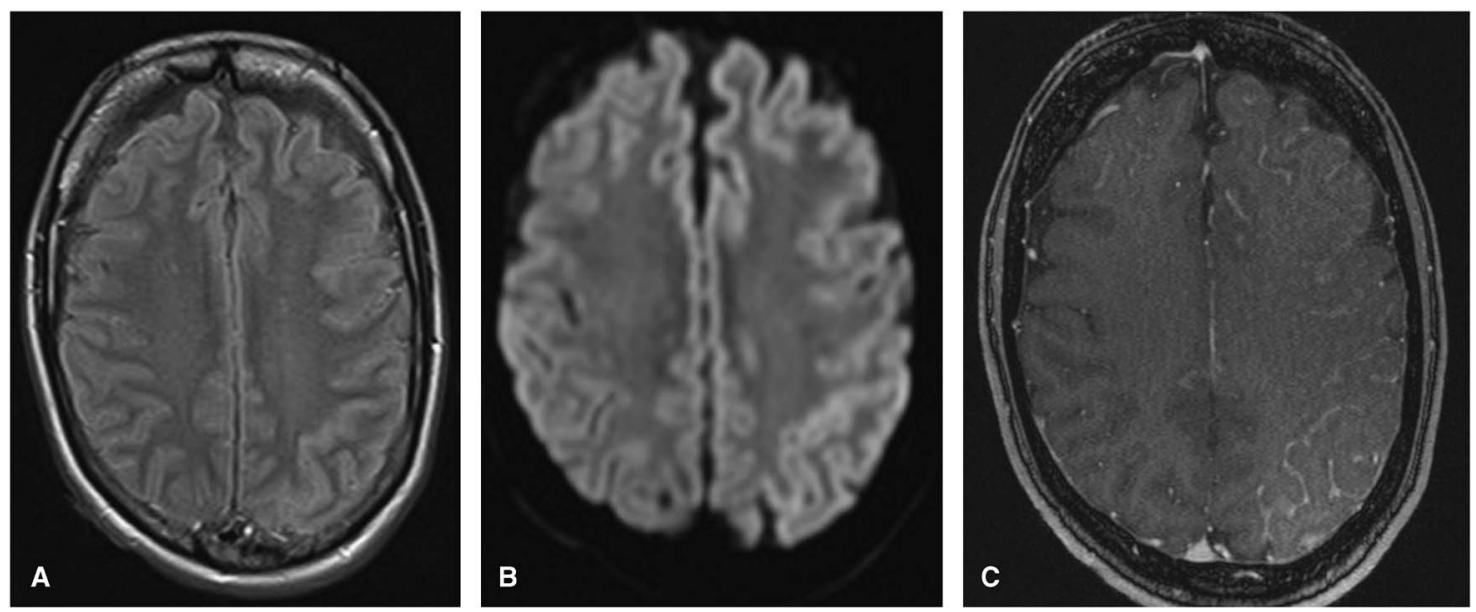

Figure 1: Brain MRI performed few hours following the intracarotid iopamidol injection. Axial fluid-attenuated inversion recovery (A) and diffusion-weighted imaging sequences $(B)$ show cortical hyperintensity in the left internal carotid artery territory. (C) Axial gadolinium-enhanced T1 weighted image show corresponding leptomeningeal enhancement involving the left frontal, parietal, and temporal lobes.

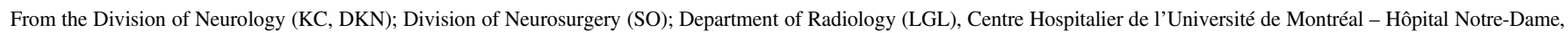
Montreal, Quebec, Canada.

Received October 6, 2016. Date of Acceptance December 12, 2016.

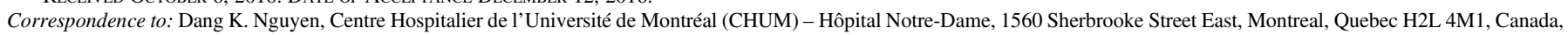
E-mail: d.nguyen@umontreal.ca 

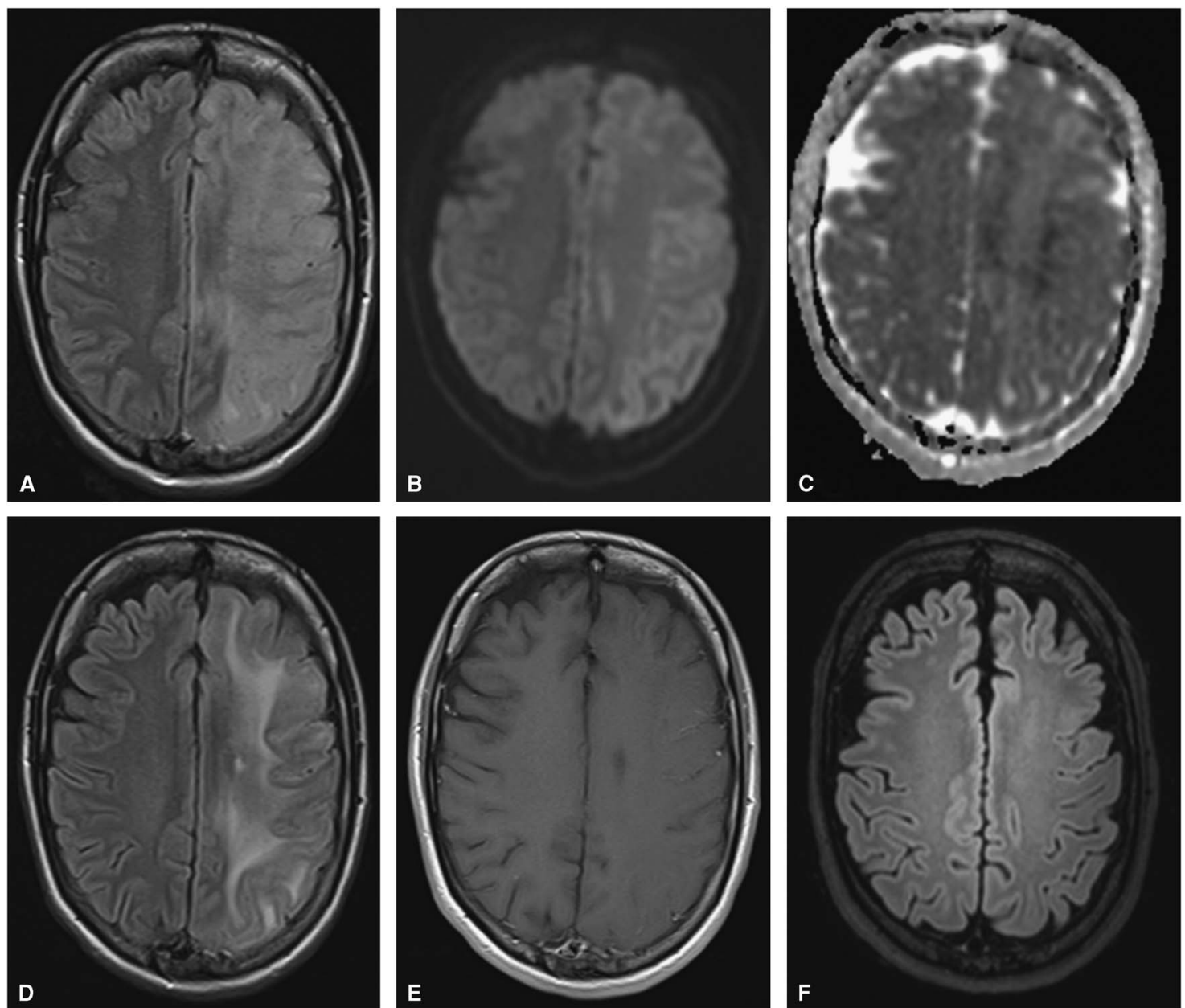

Figure 2: Axial fluid-attenuated inversion recovery $(F L A I R)(A)$, diffusion-weighted imaging $(B)$, and ADC map $(C)$ at 24 hours reveal progression of internal carotid artery territory cortical and subcortical edema and appearance of subcortical diffusion restriction. Regression of FLAIR abnormalities $(D)$ and leptomeningeal enhancement $(E)$ were observed at 1 month. Complete resolution on FLAIR imaging $(F)$ was observed at 15 months.

fluid-attenuated inversion recovery hyperintensity involving the ipsilateral hemisphere. ${ }^{2,5,6}$ Management includes exclusion of a thromboembolic event because of catheterization, early recognition, careful observation, and monitoring until self-resolution.

\section{Disclosures}

The authors do not have anything to disclose.

\section{STATEMENT OF AUTHORSHIP}

$\mathrm{KC}$ wrote the first draft of this article and approved the final manuscript as submitted. SO conceptualized and designed the case report, reviewed and revised the article for important intellectual content, and approved the final manuscript as submitted. LGL collected data, reviewed and revised the article for important intellectual content, and approved the final manuscript as submitted. DKN conceptualized and designed the case report, collected data, reviewed and revised the article for important intellectual content, and approved the final manuscript as submitted. All authors are in agreement to be accountable for all aspects of the work in ensuring that questions related to the accuracy or integrity of any part of the work are appropriately investigated and resolved.

\section{REFERENCES}

1. Loddenkemper T, Morris HH, Möddel G. Complications during the Wada test. Epilepsy Behav. 2008;13:551-3.

2. Leong S, Fanning NF. Persistent neurological deficit from iodinated contrast encephalopathy following intracranial aneurysm coiling: a case report and review of the literature. Intervent Neuroradiol. 2012;18:33-41.

3. Guimaraens L, Vivas E, Fonnegra A, et al. Transient encephalopathy from angiographic contrast: a rare complication in neurointerventional procedures. Cardiovasc Intervent Radiol. 2010;33:383.

4. Law S, Panichpisal K, Demede M, et al. Contrast-induced neurotoxicity following cardiac catheterization. Case Rep Med. 2012:267860.

5. Zwicker JC, Sila CA. MRI findings in a case of transient cortical blindness after cardiac catheterization. Cathet Cardiovasc Intervent. 2002;57:47-9.

6. Lim KK, Radford DJ. Transient cortical blindness related to coronary angiography and graft study. Med J Aust. 2002;177:43-4. 